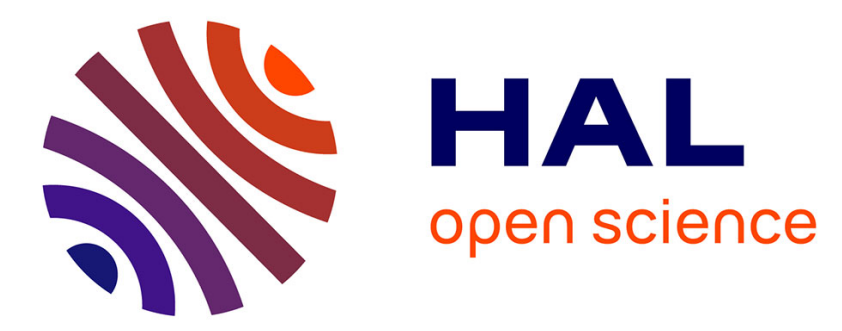

\title{
Hollow-core fibers with specific modal operation and low loss in the short-wavelength range
}

\author{
Jonas H Osório, Foued Amrani, Frédéric Delahaye, Fabio Giovanardi, Luca \\ Vincetti, Benoît Debord, Frédéric Gérôme, Fetah Benabid
}

\section{- To cite this version:}

Jonas H Osório, Foued Amrani, Frédéric Delahaye, Fabio Giovanardi, Luca Vincetti, et al.. Hollowcore fibers with specific modal operation and low loss in the short-wavelength range. International Conference on Transparent Optical Networks (ICTON), Jul 2020, Bary, Italy. hal-03006948

\section{HAL Id: hal-03006948 https://hal.science/hal-03006948}

Submitted on 16 Nov 2020

HAL is a multi-disciplinary open access archive for the deposit and dissemination of scientific research documents, whether they are published or not. The documents may come from teaching and research institutions in France or abroad, or from public or private research centers.
L'archive ouverte pluridisciplinaire $\mathbf{H A L}$, est destinée au dépôt et à la diffusion de documents scientifiques de niveau recherche, publiés ou non, émanant des établissements d'enseignement et de recherche français ou étrangers, des laboratoires publics ou privés. 


\title{
Hollow-core fibers with specific modal operation and low loss in the short-wavelength range
}

\author{
Jonas H. Osório ${ }^{1}$, Foued Amrani ${ }^{1,2}$, Frédéric Delahaye ${ }^{1,2}$, Fabio Giovanardi ${ }^{3}$, Luca Vincetti ${ }^{3}$, Benoît \\ Debord $^{1,2}$, Frédéric Gérôme ${ }^{1,2}$, Fetah Benabid ${ }^{1,2}$ \\ ${ }^{I}$ GPPMM Group, XLIM Research Institute, CNRS UMR 7252, University of Limoges, Limoges, France \\ ${ }^{2}$ GLOphotonics, 123 Avenue Albert Thomas, Limoges, France \\ 3Department of Engineering "Enzo Ferrari," University of Modena and Reggio Emilia, Modena, Italy \\ e-mail:f.benabid@xlim.fr
}

\begin{abstract}
We report on inhibited-coupling hollow-core optical fibers exhibiting specific modal operation and low loss in the short-wavelength range. We show that judicious choice of the fiber cladding structure allows obtaining fibers that favor the propagation of higher-order modes and fibers which combine effective single-mode operation and low loss. Moreover, we report on recent improvements in hollow-core fibers with ultralow loss in the visible and ultraviolet range.
\end{abstract}

Keywords: fiber optics, hollow-core fibers, photonic-crystal fibers

\section{INTRODUCTION}

The endeavors in hollow-core photonic-crystal fibers (HC-PCFs) research have led to crucial scientific comprehension of their operation and to breakthroughs both on the hollow-core fiber technology itself and on the application of these fibers to address practical needs. HC-PCFs guide light under two distinct mechanisms, namely the Photonic Bandgap (PBG) [1] and the Inhibited-Coupling (IC) [2].

The understanding of the guidance in HC-PCFs is directly tied with an in-depth knowledge of the cladding properties of fibers. This comprehension entails recognizing that, in PBG fibers, the transmission of light is possible because the core mode sits on an effective-index-frequency interval where cladding modes are absent. Inversely, IC fibers guide light thanks to a substantial minimization of the coupling between core and cladding modes, which is achieved by diminishing the spatial overlap between the core and cladding mode fields and by attaining an incongruity between transverse spatial phases of these modes [3, 4].

While the minimum measured loss for PBG fibers is $1.2 \mathrm{~dB} / \mathrm{km}$ around $1600 \mathrm{~nm}$ [5], the recent efforts in IC fibers development has placed these fibers as serious candidates to transgress the $0.14 \mathrm{~dB} / \mathrm{km}$ loss level measured for standard silica-core optical fibers [6]. Indeed, the hope on the potential of IC fibers to rival solid-core fibers has been enlivened with the introduction of the hypocycloid core contour concept (negative curvature) [7, 8]. It allowed lessening the attenuation values from the $\mathrm{dB} / \mathrm{m}$ level [9] to the current state-of-the-art figure of $8.5 \mathrm{~dB} / \mathrm{km}$ (measured at $1030 \mathrm{~nm}$ ) recently measured for kagome lattice fibers [10].

In recent years, one has witnessed advances in a promising IC-type fiber structure: the single-ring tubular lattice (SR-TL) HC-PCF [11]. The microstructure of the SR-TL HC-PCFs encompasses a single ring of nontouching tubes which delimits a fiber core with a hypocycloid form and no connecting nodes. Noteworthy, these characteristics benefit IC guidance and allow the accomplishment of ultralow loss figures, e.g., the values of $7.7 \mathrm{~dB} / \mathrm{km}$ at $780 \mathrm{~nm}$, reported by Debord et al. [3], and of $13.8 \mathrm{~dB} / \mathrm{km}$ at $539 \mathrm{~nm}$, described by Chafer et al. [12]. At shorter wavelengths, the attenuation of SR-TL HC-PCFs has been accounted as $150 \mathrm{~dB} / \mathrm{km}$ at $343 \mathrm{~nm}$, $75 \mathrm{~dB} / \mathrm{km}$ at $355 \mathrm{~nm}$ [12], and $100 \mathrm{~dB} / \mathrm{km}$ at $218 \mathrm{~nm}$ [13]. Indeed, as the loss of SR-TL HC-PCFs in the short wavelength range is limited by the surface scattering loss (SSL) [3], further decrease of the attenuation values in the visible and ultraviolet range should rely on the improvement of the core surface quality. Additionally, it is worth mentioning that novel IC HC-PCF designs (such as the ones based on conjoined tubes [14] and nested tubes [15]) have entailed a remarkable reduction in the attenuation values in the infrared spectral range. The nested tubes fiber design, for example, has recently been reported to have attenuation values as low as $0.28 \mathrm{~dB} / \mathrm{km}$ [15].

Here, we report on HC-PCFs presenting specific modal operation and ultralow loss in the visible and ultraviolet spectral ranges. The adequate choice of the fiber cladding structure offers the opportunity of having fibers that favor the propagation of higher-order modes and fibers which combine effective single-mode operation and low loss. Furthermore, we present the fabrication of an HC-PCF with ultralow loss in the short-wavelength range.

\section{HC-PCF STRUCTURES FOR SPECIFIC MODAL OPERATION}

An in-depth understanding of the cladding properties is vital for accomplishing optimized performances in HCPCFs. In this section, we describe two examples where a judicious choice of the fiber cladding allows obtaining fibers with unique modal operation. The first example consists of SR-TL HC-PCFs with modified azimuthal cladding tubes distribution able to favor the propagation of specific higher-order modes (HOMs) [16]. The second 
example concerns a new HC-PCF design, the hybrid kagome-tubular lattice (HKT) HC-PCF, which allows obtaining effective single-mode operation and low attenuation levels [17].

\subsection{Mode loss hierarchy modification by altering the distribution of cladding tubes in SR-TL HC-PCFs}

The concept of modifying the SR-TL HC-PCF structure to favor the propagation of specific higher-order modes relies on the alteration of the confinement loss (CL) hierarchy of the modes via adequate modification of the azimuthal distribution of the lattice tubes. This alteration entails enlargement of the spacing between selected tubes and implies higher leakage of the fundamental mode, whereas weakly affecting the CL of the HOMs. [16]

Figure 1a exposes two fiber designs (FD) that illustrate the concept of CL hierarchy modification: an SR-TL HC-PCF with homogeneous spacing between the lattice tubes (FD\#1) and an HC-PCF with larger spacing between the lattice tubes at $180^{\circ}$ (FD\#2). Likewise, Figure 1a presents the simulated CL of representative modes. In FD\#1, the fundamental mode ( $\mathrm{LP}_{01}$-like) is the lowest loss one. In contrast, in FD\#2, the larger spacing between the tubes at $180^{\circ}$ produces supplementary leakage of it and causes a pronounced augmentation of its CL levels. The $\mathrm{LP}_{11 \mathrm{a}^{-}}$ like mode CL, instead, is feebly touched, assuming similar figures in both FD\#1 and FD\#2. Hence, the LP11a-like mode becomes the lowest loss one in FD\#2. This concept can be extended towards obtaining an FD that favors the propagation of other HOMs (e.g., $\mathrm{LP}_{21}$-like), as described in [16].

Figure $1 \mathrm{~b}$ shows a fiber that was drawn to illustrate the concept described in the last paragraph. As it can be observed in Figure 1b, the fiber presents wider inter-tubes gaps oriented at $180^{\circ}$. To account for the $\mathrm{LP}_{11}$ mode loss, one has optimized the light coupling in order to obtain an $\mathrm{LP}_{11}$-like profile at the fiber output. In sequence, a cutback measurement was performed. The loss of the $\mathrm{LP}_{11}$ was measured to be $200 \mathrm{~dB} / \mathrm{km}$ around $1 \mu \mathrm{m}$. Additionally, mode transformation experiments were conducted. They showed the capability of this FD to provide atypical intensity profiles at the fiber output by suitably coupling a superposition of the $\mathrm{LP}_{01}$ and $\mathrm{LP}_{11}$ modes and by tuning the input light polarization. Figure 1c shows the generated profiles. Remarkably, the lobes in the fiber output profile have orthogonal polarization [16]. The yields of this study are expected to be employed in atom and nonlinear optics applications.
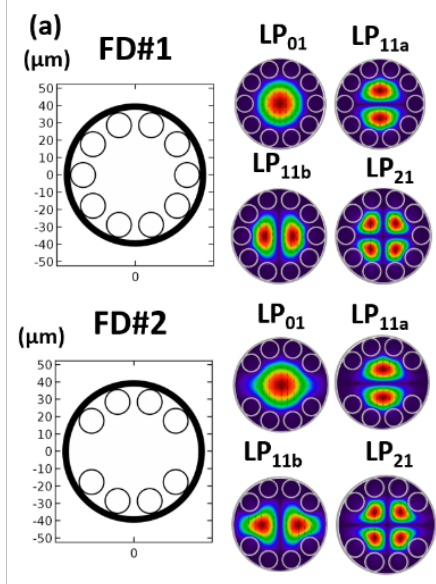

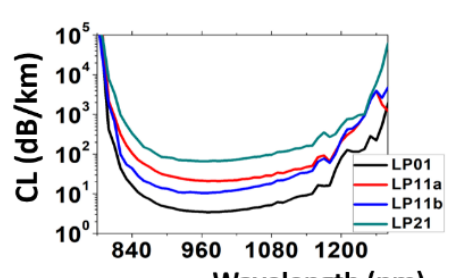

Wavelength $(\mathrm{nm})$

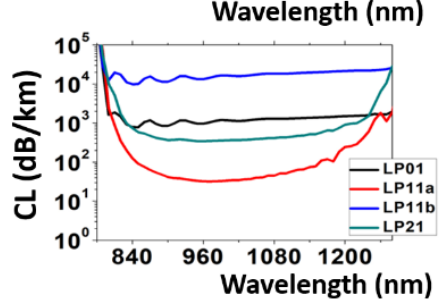

(b)
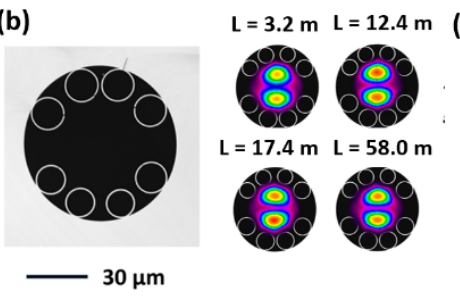

(c)

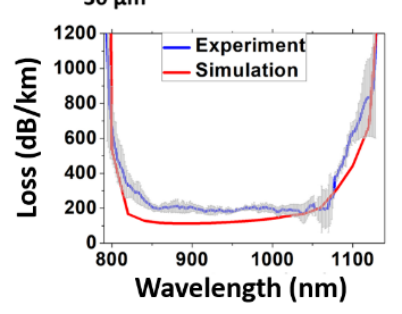

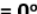

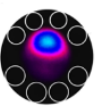

$\theta=45^{\circ}$

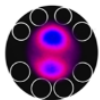

$\theta=90^{\circ}$

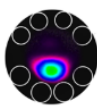

$\theta=135^{\circ}$

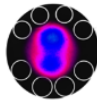

$\theta=180^{\circ}$

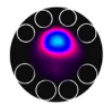

Figure 1. (a) Fiber designs, representative mode profiles, and their CL. (b) Fabricated fiber and loss measurement. (c) Fiber output profiles for different input polarization angles.

\subsection{Hybrid kagome-tubular (HKT) HC-PCF for effective single-mode operation and low loss}

A second example of how adequately designing IC fibers can entail structures with outstanding performances is represented by the HKT HC-PCF. This novel design associates kagome and tubular lattices for attaining low loss and effective single-mode operation. The ideal HKT HC-PCF structure is presented in Figure 2a. The inner cladding is formed by a lattice of six tubes and is surrounded by a kagome lattice (the outer cladding). The sixtubes lattice allows filtering the $\mathrm{LP}_{11}$ mode via resonant coupling between the latter and the $\mathrm{LP}_{01}$ mode in the cladding tubes. This property entails effective single-mode operation in the fiber since the $\mathrm{LP}_{11}$ mode is usually the foremost contaminating mode of the fiber modal content $[18,19]$.

The potential of the HKT HC-PCF to provide ultralow loss can be observed in Figure 2b, where the CL of a jacketless tubular fiber (FD\#3), an SR-TL HC-PCF (FD\#4), a kagome fiber (FD\#5) and an ideal version of the HKT HC-PCF (FD\#6) are presented as a function of the normalized frequency, $F=\left(\frac{2 t}{\lambda}\right) \sqrt{n_{g}^{2}-1}$, where $t$ represents the thickness of the cladding tubes, $\lambda$ is the wavelength, and $n_{g}$ is the refractive index of glass. In the ideal version of the HKT HC-PCF, the tubular and kagome claddings have no physical connection. Even though this structure is not feasible, it allows identifying the potential of the HKT HC-PCF design to achieve a dramatic reduction of CL values, and it is of great academic interest. Figure $2 b$ shows that the CL values of the HKT HC- 
PCF are significantly lower than the ones simulated for the other FDs. We see that this difference reaches five orders of magnitude in the second-order transmission band. Moreover, the CL of the ideal HKT HC-PCF is significantly lower than the loss of standard solid-core fibers, represented as a dashed line in Figure $2 \mathrm{~b}$.

While the ideal HKT HC-PCF is not practically attainable, we propose a realizable version of it by including thin tubes to connect the inner and outer claddings. The addition of the attaching tubes upsets the CL calculated for the ideal HKT HC-PCF yet entailing CL values as low as $1.46 \times 10^{-2} \mathrm{~dB} / \mathrm{km}$ [17]. This loss level is still significantly lower than the CL of other FD explored in the literature based on conjoined and nested tubes.

Figure $2 \mathrm{c}$ presents the experimentally achieved HKT HC-PCF. The inner cladding tubes have a thickness of $1.27 \mu \mathrm{m}$, and the fiber core diameter measures $37.1 \mu \mathrm{m}$. The ratio between the core and cladding tubes diameters ( $\left.D_{\text {tubes }} / D_{\text {core }}\right)$ was optimized during fiber fabrication to provide optimum coupling between the LP 11 mode guided in the core and the fundamental mode of the cladding tubes. In the fabricated fiber, $D_{\text {tubes }} / D_{\text {core }}=0.62$. Figure $2 \mathrm{c}$ exposes the results of a cutback measurement, where a minimum loss figure of $1.6 \mathrm{~dB} / \mathrm{km}$ at $1050 \mathrm{~nm}$ could be measured. Figure $2 \mathrm{~d}$ also presents the simulated CL and total loss $(\mathrm{TL}=\mathrm{CL}+\mathrm{SSL}$, where SSL is the surface scattering loss), which were calculated by taking into account the experimental structure. Good similarity is observed between experimental and simulated data.

Finally, we performed $\mathrm{S}^{2}$ measurements to assess the fiber modal content. For optimized light launching conditions, the measurements show a HOM extinction ratio of $47 \mathrm{~dB}$ for a $10 \mathrm{~m}$-long fiber (Figure 2e shows the $\mathrm{S}^{2}$ measurement trace). Remarkably, the HKT HC-PCF entails low loss combined with effective single-mode operation. Further details on this research can be found in [17].
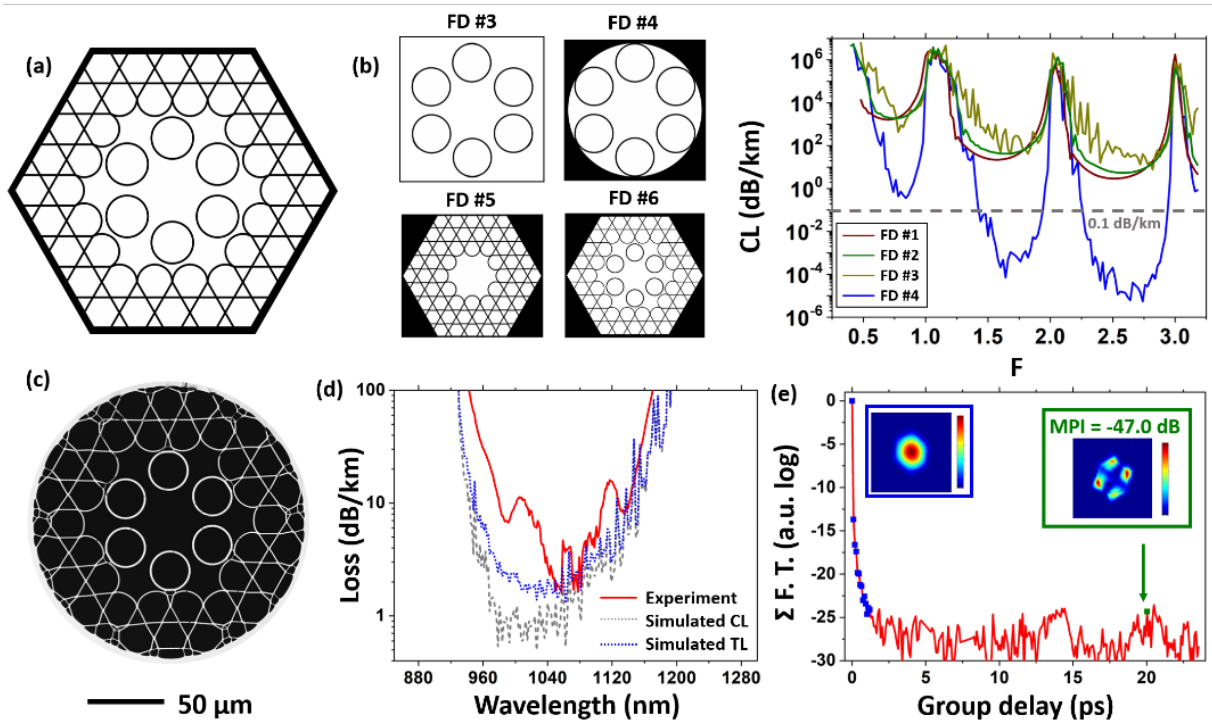

Figure 2. (a) HKT HC-PCF structure. (b) CL as a function of FD for tubular, kagome, and hybrid FD. (c) Experimental HKT HC-PCF. (d) Measured loss and simulated CL and TL. (e) $S^{2}$ measurement results for an HKT HC-PCF with length $10 \mathrm{~m}$. MPI: multi-path interference.

\section{HC-PCFS WITH LOW LOSS IN THE SHORT-WAVELENGTH RANGE}

As SR-TL HC-PCFs work on the large-pitch regime and provide light guidance with very low overlap between the guided mode and the core contour [3, 4], they emerge as an up-and-coming platform for transmitting visible and ultraviolet light. As mentioned before, the reduction of the loss values in the short-wavelength range should count on the amelioration of the core surface quality since the SSL is the dominating loss mechanism.

Thus, by revisiting the usual HC-PCF fabrication technique, we improved on the quality of the core surface of our fibers and attained remarkable low loss figures in the short wavelength range. Here, we present one of these fibers, whose cross-section is shown in Figure 3a. The fiber consists of an SR-TL HC-PCF with a lattice of 8 tubes. The fiber core diameter is $27 \mu \mathrm{m}$, and the spacing between the lattice tubes ranges from $2.1 \mu \mathrm{m}$ to $4.7 \mu \mathrm{m}$. Cutback measurements were performed, and minimum loss figures of $6.5 \mathrm{~dB} / \mathrm{km}$ at $492 \mathrm{~nm}, 9.7 \mathrm{~dB} / \mathrm{km}$ at $369 \mathrm{~nm}$, and $61.0 \mathrm{~dB} / \mathrm{km}$ at $292 \mathrm{~nm}$ were measured (Figure 3b). Noteworthy, these values represent the new state-of-the loss at these wavelengths. It is worth mentioning that the cutback measurement for wavelengths larger than $400 \mathrm{~nm}$ was performed by using a supercontinuum source and an optical spectrum analyzer. For wavelengths shorter than $400 \mathrm{~nm}$, the cutback measurement was performed by using a plasma lamp, and a spectrometer measured the transmitted light. Careful alignment was undertaken to account for the loss figures in the ultraviolet range.

Moreover, it is worth remarking that the loss figures of the fiber described herein are below the fundamental Rayleigh scattering limit of bulk silica, as represented in Figure 3b. Furthermore, it is notable that this new generation fibers for the short wavelength range allows addressing important practical needs as, for example, the 
development of light sources in the UV range. An example has been recently explored by Chafer et al. [20, 21], who studied a UV Raman comb pumped by a $355 \mathrm{~nm}$ laser.

Supplementary results and details on this research will be described in a forthcoming publication.

(a)

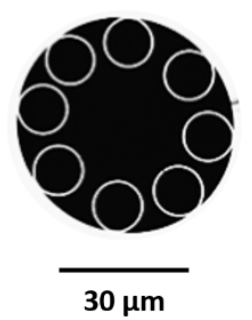

(b)

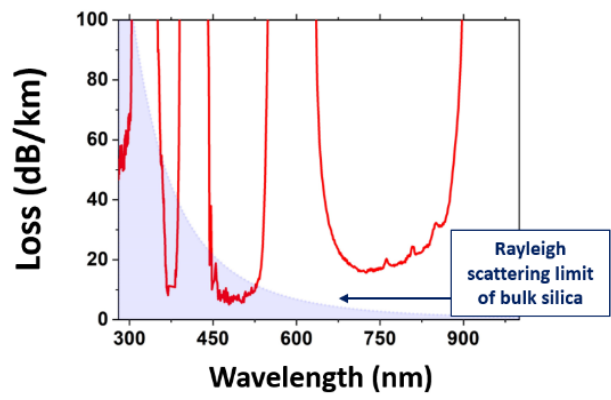

Figure 3. (a) Cross-section of the SR-TL HC-PCF and (b) cutback measurement results.

\section{CONCLUSIONS}

In this manuscript, we described recent developments on HC-PCFs displaying distinctive modal operation and ultralow loss in the short-wavelength range. We emphasized that the accomplishment of fibers with such distinctive modal characteristics relies on a careful choice of the fiber cladding structure. In this context, we exposed the concept of altering the fiber loss hierarchy by suitably changing the azimuthal position of the cladding tubes in SL-TL HC-PCFs. Additionally, we presented a new fiber design, the HKT HC-PCF, which associates kagome and tubular lattices to achieve effective single-mode operation and low attenuation values. Additionally, we reported on the experimental realization of the HKT HC-PCF. Finally, we described an SR-TL HC-PCF with ultralow loss in the short-wavelength range.

\section{ACKNOWLEDGMENTS}

This research was funded through PIA program (grant 4F) and la region de la Nouvelle Aquitaine

\section{REFERENCES}

[1] Birks, T. A. et al. "Full 2-D photonic bandgaps in silica/air structures," Electron. Lett. 31, 22, 1995.

[2] Couny, F. et al. "Generation and photonic guidance of multi-octave optical-frequency combs," Science 318, 2007.

[3] Debord, B. et al. "Ultralow transmission loss in inhibited-coupling guiding hollow fibers," Optica 4, 209-217, 2017.

[4] Debord, B. et al. "Hollow-core fiber technology: the rising of 'gas photonics", Fibers 7(2), 16, 2019.

[5] Roberts, P. J. et al. "Ultimate low loss of hollow-core photonic crystal fibers," Opt. Express 13, 1, 236-244, 2005.

[6] Tamura, Y. et al., "The first 0.14-dB/km loss optical fiber and its impact on submarine transmission," J. Light. Technol. 36, 44-49, 2018.

[7] Wang, Y. et al. "Low loss broadband transmission in optimized core-shape Kagome hollow-core PCF," in CLEO (OSA, 2010), paper CPDB4.

[8] Wang, Y. Y. et al. "Low loss broadband transmission in hypocycloid-core Kagome hollow-core photonic crystal fiber," Opt. Lett. 36, 669-671, 2011.

[9] Benabid, F. et al. "Stimulated Raman scattering in hydrogen-filled hollow-core photonic crystal fiber," Science 298, 5592, 399-402, 2002.

[10] Maurel, M. et al. "Optimized inhibited-coupling Kagome fibers at Yb-Nd:Yag (8.5 dB/km) and Ti:Sa (30 dB/km) ranges," Opt. Lett. 43, 1598-1601, 2018.

[11] Pryamikov, A. D. et al. "Demonstration of a waveguide regime for a silica hollow-core microstructured optical fiber with a negative curvature of the core boundary in the spectral region $>3.5 \mu \mathrm{m}$," Opt. Express 19, 1441-1448 (2011).

[12] Chafer, M. et al. "1 km hollow-core fiber with loss at the silica Rayleigh limit in the green spectral region," IEEE Photon. Technol. Lett. 31, 685-688, 2019.

[13] Yu, F. et al. "Single-mode solarization-free hollow-core fiber for ultraviolet pulse delivery," Opt. Express 26, 2018.

[14] Gao, S. et al., "Hollow-core conjoined-tube negative-curvature fibre with ultralow loss," Nat. Commun., 9, $2828,2018$.

[15] Jasion, G. T. et al. "Hollow core NANF with $0.28 \mathrm{~dB} / \mathrm{km}$ attenuation in the C and L bands," in OFC Postdeadline Papers 2020, (OSA, 2020), paper Th4B.4.

[16] Osório, J. H. et al. "Tailoring modal properties of inhibited-coupling fibers by cladding modification," Sci. Rep., 9:1376, 2019

[17] Amrani, F. et al. "Low-loss single-mode hybrid-lattice hollow-core fiber" arXiv:2006.06375, 2020.

[18] Bradley, T. D. et al. "Optical properties of low loss $(70 \mathrm{~dB} / \mathrm{km})$ hypocycloid-core kagome hollow core photonic crystal fiber for Rb and Cs based optical applications," J. Light. Technol. 31, 2752-2755 (2013).

[19] Uebel, P. et al. "Broadband robustly single-mode hollow-core PCF by resonant filtering of higher-order modes," Opt. Lett. 41, 2016.

[20] Chafer, M. et al. "UV-DUV source based on IC-HCPCF filled with Hydrogen," in Laser Congress 2019 (ASSL, LAC, LS\&C) (OSA, 2019), paper ATh4A.2.

[21] Chafer, M. et al. "355 nm-laser pumped hydrogen UV Raman comb," in 2019 CLEO Europe (OSA 2019), paper cj 35. 